\title{
The performance of active noise control systems on ground with two parallel reflecting surfaces
}

Jiaxin Zhong, Baicun Chen, Jiancheng Tao, and Xiaojun Qiu

Citation: The Journal of the Acoustical Society of America 147, 3397 (2020); doi: 10.1121/10.0001227

View online: https://doi.org/10.1121/10.0001227

View Table of Contents: https://asa.scitation.org/toc/jas/147/5

Published by the Acoustical Society of America

\section{ARTICLES YOU MAY BE INTERESTED IN}

A spherical expansion for audio sounds generated by a circular parametric array loudspeaker

The Journal of the Acoustical Society of America 147, 3502 (2020); https://doi.org/10.1121/10.0001261

Using empirical wavelet transform to speed up selective filtered active noise control system

The Journal of the Acoustical Society of America 147, 3490 (2020); https://doi.org/10.1121/10.0001220

Beamforming correction for the singular problem in identifying rotating sources with non-uniform directivity

The Journal of the Acoustical Society of America 147, 3151 (2020); https://doi.org/10.1121/10.0001169

Increasing the performance of active noise control systems on ground with two vertical reflecting surfaces with an included angle

The Journal of the Acoustical Society of America 146, 4075 (2019); https://doi.org/10.1121/1.5134062

A time domain decentralized algorithm for two channel active noise control

The Journal of the Acoustical Society of America 147, 3808 (2020); https://doi.org/10.1121/10.0001401

Experimental study of airfoil-rotor interaction noise by wavelet beamforming

The Journal of the Acoustical Society of America 147, 3248 (2020); https://doi.org/10.1121/10.0001209

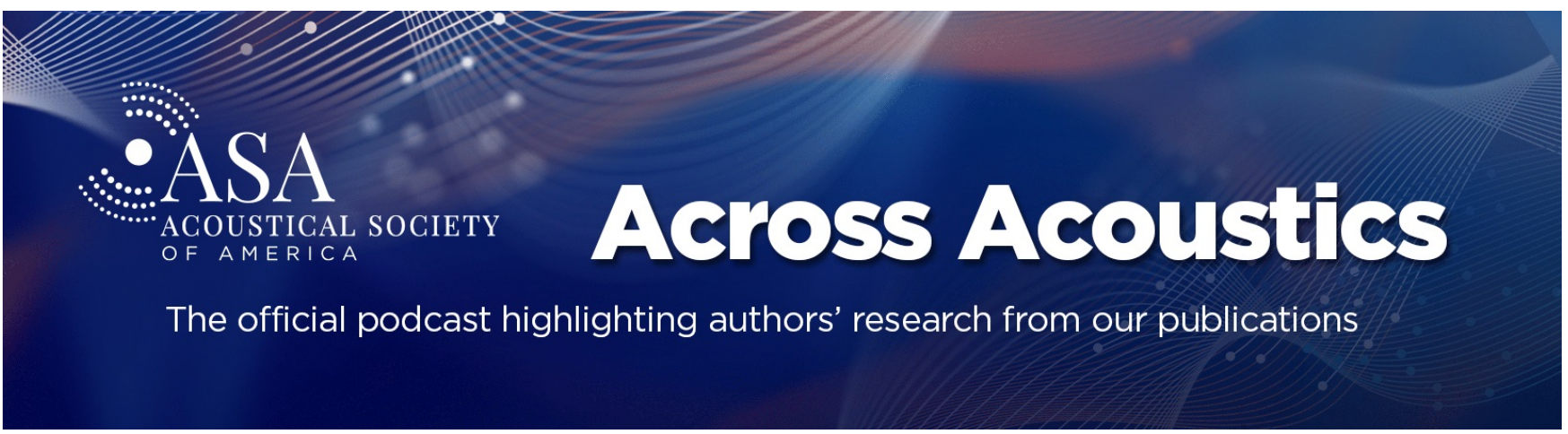




\title{
The performance of active noise control systems on ground with two parallel reflecting surfaces
}

\author{
Jiaxin Zhong, ${ }^{1, a)}$ Baicun Chen, ${ }^{2}$ Jiancheng Tao, ${ }^{2, b)}$ and Xiaojun Qiu ${ }^{1, \mathrm{c})}$ \\ ${ }^{1}$ Centre for Audio, Acoustics and Vibration, Faculty of Engineering and Information Technology, University of Technology Sydney, \\ New South Wales 2007, Australia \\ ${ }^{2}$ Key Laboratory of Modern Acoustics, Institute of Acoustics, Nanjing University, Nanjing 210093, China
}

\begin{abstract}
:
This paper investigates the performance of active noise control (ANC) systems with two reflecting surfaces that are placed vertically on ground in parallel. It employs the modal expansion method and the boundary element method to calculate the noise reduction of the systems with infinitely large and finite size reflecting surfaces, respectively. Both experimental and simulation results show that the noise reduction of the system can be significantly increased after optimizing the surface separation distance and their locations with the sound sources. It is found that the sound radiation of the primary source can be completely reduced in principle if the surface interval is less than half the wavelength and the source line is perpendicular to the surfaces for infinitely large reflecting surfaces. Even with finite size ones, the noise reduction performance improvement is still significant compared with those without any reflecting surfaces. For example, for an ANC system with a source distance of $0.074 \mathrm{~m}$, experiments achieve an improvement of $8.6 \mathrm{~dB}$ at $800 \mathrm{~Hz}$ where two $0.2 \mathrm{~m} \times 0.2 \mathrm{~m}$ parallel reflecting surfaces are placed with a distance of $0.15 \mathrm{~m}$ around the system on ground. The mechanisms for the performance improvement are discussed.

(C) 2020 Acoustical Society of America. https://doi.org/10.1121/10.0001227
\end{abstract}

(Received 19 November 2019; revised 8 March 2020; accepted 21 April 2020; published online 12 May 2020)

[Editor: Wonkyu Moon]

Pages: 3397-3407

\section{INTRODUCTION}

The noise reduction performance of active noise control (ANC) systems on ground is affected by reflecting surfaces around the system due to the change of the sound source radiation resistance caused by the surfaces. ${ }^{1}$ The effects of reflecting surfaces on ANC systems have attracted much attention, and several cases have been studied, which include an infinitely large reflecting surface, ${ }^{2-5}$ a finite size one, ${ }^{6}$ two vertically placed ones that are perpendicular to each other (edge), ${ }^{7,8}$ and two vertically placed ones with an arbitrary included angle (wedge). ${ }^{9}$

The sound power output of point monopoles in the presence of one reflecting surface has been investigated with the image source method. ${ }^{10,11}$ Based on this method, the effects of one reflecting surface on both single channel and multi-channel ANC systems were studied. For single channel ANC systems, the noise reduction increases when the primary and secondary sources are placed along a line perpendicular to the reflecting surface. ${ }^{5}$ For multi-channel ANC systems with the primary source on the reflecting surface, the noise reduction can be maximally increased if the secondary sources are placed as far apart from each other and the ground as possible. ${ }^{4}$ The mechanism is that the additional reflecting surfaces can change the radiation properties of the sound sources to enhance the performance

\footnotetext{
a) ORCID: 0000-0002-9972-8004.

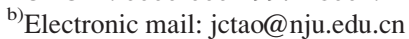

${ }^{c)}$ ORCID: 0000-0002-5181-1220.
}

of ANC systems. By using a finite size reflecting surface vertical to the ground, the noise reduction can be further increased. $^{6}$

For ANC systems near two reflecting surfaces with the right included angle, the noise reduction of the system has been investigated. Numerical results show that higher reduction of the ANC system can be achieved compared with a case with only one reflecting surface by optimizing the directions and locations between the sources and surfaces. ${ }^{7}$ The case of two reflecting surfaces with an arbitrary included angle has been investigated, and research found that higher noise reduction can be obtained at the optimal angle of about $120^{\circ} .9$ The experiment results show the noise reduction can be further increased by $3.6 \mathrm{~dB}$ compared to a case with only one reflecting surface. ${ }^{9}$

In some applications, two parallel reflecting surfaces can be used around ANC systems. For example, parallel reflecting surfaces can be introduced directly around a small system. For large noise sources like electrical transformers, a vertical radiation surface can be divided into several small ones by placing a set of parallel barriers perpendicular to the large surface so that the noise radiation of the large source can be decoupled and controlled in each pair of barriers. ${ }^{8}$ Louvers are often used for noise control, where a set of angled slats or flat strips parallel to each other are fixed or hung at regular intervals in the opening to allow air or light to pass through. ${ }^{12,13}$ For sound transmitted through the louvers to outside, active control can be applied between the parallel slats. The effects of two parallels of reflecting 
surfaces on the noise reduction performance of ANC systems is unknown at present.

The power output of point monopoles inside two parallel reflecting surfaces has been analyzed by using the image source method. ${ }^{14,15}$ To overcome the slow convergence speed of the image source method, the layered media theory of sound propagation has been developed by representing the radiation of point monopoles inside a one-layer medium bounded on two sides by plane parallel boundaries with the summation of the normal modes. ${ }^{16}$ When the size of the reflectors is finite, no analytical solutions are available, but the sound field can be calculated using numerical methods such as the boundary element method (BEM). ${ }^{17}$

This paper investigates the feasibility of increasing the noise reduction performance of ANC systems by introducing two reflecting surfaces that are placed vertically on ground in parallel. First, the noise reduction of the ANC system inside two infinitely large reflecting surfaces is analyzed with the normal mode theory inside a one-layer medium, and then the effects of the included angle between the source line and the normal line of the surfaces as well as the surface interval are studied. The noise reduction of the system for the finite size reflecting surfaces are investigated with the BEM, and the mechanisms for the performance improvement are discussed. Finally, the experimental results serve to validate the analytical and simulation results.

\section{THEORY}

For a single channel ANC system on ground shown in Fig. 1, the distance between the primary and secondary sources is $d$, and the separation distance between the two reflecting surfaces placed vertically in parallel with dimensions of $w \times h$ is $D$. The included angle between the source line and the normal line of the surfaces (i.e., the line perpendicular to the parallel surfaces) is denoted by $\theta$. The ground and both sides of reflecting surfaces are assumed to be perfectly reflective throughout the paper.

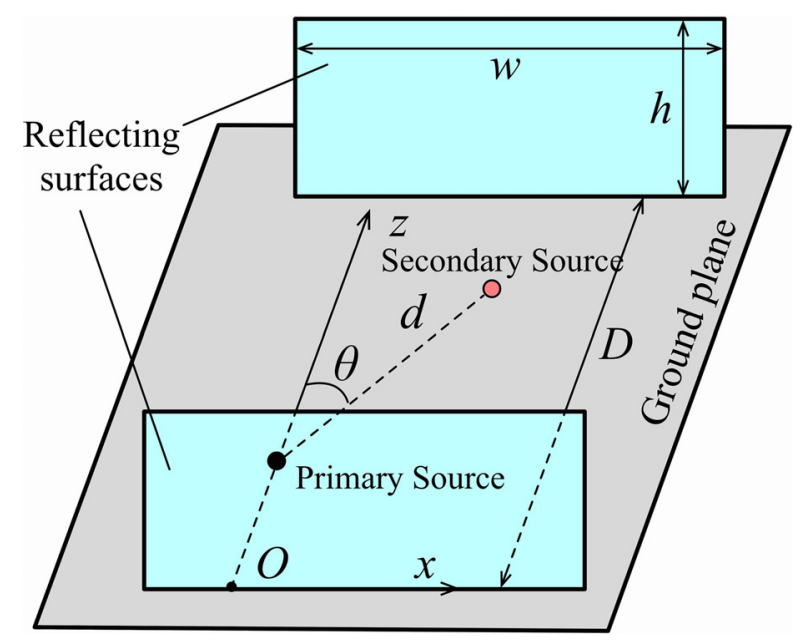

FIG. 1. (Color online) Schematic diagram of an ANC system with two reflecting surfaces placed vertically on ground in parallel.
A cylindrical coordinate system $(\rho, \varphi, z)$ is established with the origin, $O$, located at the projection of the primary source on the nearer reflecting surface shown in Fig. 1. The $z$ axis direction is perpendicular to the surfaces and points to the other reflecting surface. The ground plane is at $y=0$, so the location of the image source from the ground for a sound source located at $(\rho, \varphi, z)$ is $(\rho,-\varphi, z)$. When the source is on the ground plane $(y=0)$, the point monopole and its image coincide. In this paper, both sources are assumed to be on ground to simplify the problems in order to focus on the effects of the two parallel reflecting surfaces on ANC systems, so the locations of the primary and the secondary sources are $\mathbf{r}_{\mathrm{p}}=\left(\rho_{\mathrm{p}}, 0, z_{\mathrm{p}}\right)$ and $\mathbf{r}_{\mathrm{s}}=\left(\rho_{\mathrm{s}}, 0, z_{\mathrm{s}}\right)$, respectively.

The infinitely large reflecting surfaces are considered first because its sound field can be solved analytically. ${ }^{16}$ The sound pressure at the point $\mathbf{r}=(\rho, \varphi, z)$ generated by a point monopole at $\mathbf{r}_{0}=\left(\rho_{0}, \varphi_{0}, z_{0}\right)$ inside two infinitely large reflecting surfaces that are placed vertically in parallel can be calculated by (see Chap. 5 in Ref. 16)

$$
\begin{aligned}
p\left(\mathbf{r} ; \mathbf{r}_{0}\right)= & \frac{\rho_{\text {air }} \omega k q_{\text {src }}}{4 \pi} \sum_{m=0}^{\infty} \sum_{n=0}^{\infty} \frac{\pi}{k D} \varepsilon_{m} \varepsilon_{n} \cos \left(n \pi \frac{z}{D}\right) \\
& \times \cos \left(n \pi \frac{z_{0}}{D}\right) \cos \left[m\left(\varphi-\varphi_{0}\right)\right] \\
& \times \mathrm{J}_{m}\left(\sqrt{k^{2}-(n \pi / D)^{2}} \rho_{<}\right) \\
& \times \mathrm{H}_{m}\left(\sqrt{k^{2}-(n \pi / D)^{2}} \rho_{>}\right)
\end{aligned}
$$

where $\mathrm{j}$ is the imaginary unit, $\rho_{\text {air }}$ is the air density, $k$ is the wavenumber, $\omega$ is the angular frequency, $q_{\text {src }}$ is the source strength, $\rho_{<}=\min \left(\rho, \rho_{0}\right), \rho_{>}=\max \left(\rho, \rho_{0}\right), \varepsilon_{n}$ is the Neumann factor [i.e., $\varepsilon_{n}=1(n=0)$ and $\varepsilon_{n}=2(n=1,2,3$, $\ldots)], \mathrm{J}_{m}(\cdot)$ is the Bessel function of the first kind of order $m$, and $\mathrm{H}_{m}(\cdot)$ is the Hankel function of the first kind of order $m$. When the two sources are on the ground shown in Fig. 1, the sound pressure produced by each source is two times the sound pressure computed by Eq. (1).

The sound radiation power of a single ANC channel system consisting of one primary source and one secondary source can be formulated as ${ }^{18,19}$

$$
W=A\left|q_{\mathrm{s}}\right|^{2}+q_{\mathrm{s}}^{*} b+b^{*} q_{\mathrm{s}}+c,
$$

where $q_{\mathrm{s}}$ is the complex source strength of the secondary source, * denotes complex conjugation, $A=R_{\mathrm{S}} / 2, R_{\mathrm{S}}$ is the self-radiation resistance of the secondary source, $b=q_{\mathrm{p}} R_{\mathrm{ps}} /$ $2, R_{\mathrm{ps}}$ is the mutual radiation resistance between the primary source and the secondary source, $c=\left|q_{\mathrm{p}}\right|^{2} R_{\mathrm{p}} / 2$, and $q_{\mathrm{p}}$ and $R_{\mathrm{p}}$ are the complex source strength and the self-radiation resistance of the primary source, respectively. Equation (2) is obtained based on the fundamental equations in homogenous media (Sec. 9.11 of Ref. 19) where the free field condition is not needed, so it is valid for the sound source in any acoustic environments. ${ }^{18}$

The resistances can be calculated with the pressure obtained by using Eq. (1) as ${ }^{18,19}$ 


$$
\begin{aligned}
& R_{\mathrm{p}}=\operatorname{Re}\left[p\left(\mathbf{r}_{\mathrm{p}} ; \mathbf{r}_{\mathrm{p}}\right) / q_{\mathrm{p}}\right], \\
& R_{\mathrm{s}}=\operatorname{Re}\left[p\left(\mathbf{r}_{\mathrm{s}} ; \mathbf{r}_{\mathrm{s}}\right) / q_{\mathrm{s}}\right], \\
& R_{p s}=\operatorname{Re}\left[p\left(\mathbf{r}_{\mathrm{p}} ; \mathbf{r}_{s}\right) / q_{s}\right],
\end{aligned}
$$

where $\operatorname{Re}[\cdot]$ denotes the real part of the quantity inside the square brackets.

After obtaining the optimal secondary source strength, ${ }^{18,19}$

$$
q_{\mathrm{s}, \mathrm{opt}}=-\frac{R_{\mathrm{ps}}}{R_{\mathrm{s}}} q_{\mathrm{p}}
$$

the sound radiation power under optimal control is

$$
W_{\mathrm{opt}}=\frac{1}{2}\left|q_{\mathrm{p}}\right|^{2}\left(R_{\mathrm{p}}-\frac{R_{\mathrm{ps}}^{2}}{R_{\mathrm{s}}}\right) .
$$

The noise reduction is defined as

$$
\mathrm{NR} \equiv-10 \lg \left(\frac{W_{\mathrm{opt}}}{W_{0}}\right)
$$

where the sound radiation power of the primary source on ground $W_{0}=\left(\rho_{\text {air }} \omega k\left|q_{\mathrm{p}}\right|^{2}\right) /(4 \pi)$ is used as the reference. This defined noise reduction is $0 \mathrm{~dB}$ without active noise control if there are no additional reflecting surfaces around the system. For a constant volume primary source on ground, its sound radiation power (without ANC) varies after introducing reflecting surfaces near it. For example, its sound radiation power is increased by $3 \mathrm{~dB}$ when an infinitely large reflecting surface is introduced near it at the low frequency. ${ }^{10}$ Therefore, the noise reduction defined by Eq. (8) can be nonzero (or even negative) without ANC when reflecting surfaces are placed around it.

When the primary and secondary sources are located on ground at a distance of $d$ without any reflecting surfaces, the noise reduction is known as ${ }^{19}$

$$
\mathrm{NR}_{\text {gnd }}=-10 \lg \left(1-\left[\frac{\sin (k d)}{k d}\right]^{2}\right) .
$$

In the following paragraphs, infinitely large reflecting surfaces are considered first. The optimal included angle between the source line and the normal line of the surfaces is investigated. Then the effects of the surface interval on the noise reduction performance of ANC systems are discussed. Finally, the finite size reflecting surfaces are considered.

The self-radiation resistances of the primary and secondary sources and the mutual radiation resistance between them can be simplified after substituting Eq. (1) into Eqs. (3)-(5) as (see the Appendix)

$$
\begin{aligned}
& R_{\mathrm{p}}=\frac{\rho_{\text {air }} \omega k}{2 \pi} \sum_{n=0}^{\lfloor k D / \pi\rfloor} \frac{\pi}{k D} \varepsilon_{n} \cos ^{2}\left(\frac{z_{\mathrm{p}}}{D} n \pi\right), \\
& R_{s}=\frac{\rho_{\text {air }} \omega k}{2 \pi} \sum_{n=0}^{\lfloor k D / \pi\rfloor} \frac{\pi}{k D} \varepsilon_{n} \cos ^{2}\left(\frac{z_{\mathrm{s}}}{D} n \pi\right),
\end{aligned}
$$

$$
\text { and } \begin{aligned}
R_{\mathrm{ps}}= & \frac{\rho_{\mathrm{air}} \omega k}{2 \pi} \sum_{n=0}^{\lfloor k D / \pi\rfloor} \frac{\pi}{k D} \varepsilon_{n} \cos \left(\frac{z_{\mathrm{p}}}{D} n \pi\right) \\
& \times \cos \left(\frac{z_{\mathrm{s}}}{D} n \pi\right) \mathrm{J}_{0}\left(\sqrt{1-\left(\frac{n \pi}{k D}\right)^{2}} k d \sin \theta\right),
\end{aligned}
$$

respectively, where $D \geq d$ to ensure the two sources are inside the two surfaces when $\theta=0$ and $\lfloor\cdot\rfloor$ represents a rounded down version of the quantity inside it. The upper bound of the summation is $\lfloor k D / \pi\rfloor$ because all the modes such that $n>k D / \pi$ (i.e., $D<\lambda / 2$ where $\lambda$ is the wavelength) are evanescent waves, which do not contribute to the radiation resistance (see the Appendix for the details).

The optimal secondary source strength and the maximal noise reduction of the ANC system when $D<\lambda / 2$ can be derived by substituting Eqs. (10) and (11) into Eqs. (6) and (7) as

$$
\begin{aligned}
& q_{\mathrm{s}, \mathrm{opt}}=-q_{\mathrm{p}} \mathrm{J}_{0}(k d \sin \theta), \\
& \text { and } \mathrm{NR}=-10 \lg \left(\frac{\pi}{k D}\left[1-\mathrm{J}_{0}^{2}(k d \sin \theta)\right]\right),
\end{aligned}
$$

respectively. According to the values of the Bessel function of order zero $\mathrm{J}_{0}(\cdot)$, Eq. (13) shows that the system noise reduction increases as the included angle $\theta$ decreases at low frequencies and approaches infinitely large when $\theta=0^{\circ}$. In such a case, the source line is perpendicular to the two reflecting surfaces.

At the optimal case, when the source line is perpendicular to the two reflecting surfaces, the mutual radiation resistance between them described by Eq. (11) can be further simplified as

$$
R_{\mathrm{ps}}=\frac{\rho_{\mathrm{air}} \omega k}{2 \pi} \sum_{n=0}^{\lfloor k D / \pi\rfloor} \frac{\pi}{k D} \varepsilon_{n} \cos \left(\frac{z_{\mathrm{p}}}{D} n \pi\right) \cos \left(\frac{z_{\mathrm{s}}}{D} n \pi\right),
$$

with the relation $\mathrm{J}_{0}(0)=1 .^{20}$

The optimal secondary source strength and the maximal noise reduction of the ANC system at $\theta=0$ can be derived similarly to Eqs. (12) and (13) as

$$
\begin{aligned}
& q_{\mathrm{s}, \mathrm{opt}}=-q_{\mathrm{p}}, \\
& \text { and } N R \rightarrow \infty
\end{aligned}
$$

respectively.

The noise reduction in Eq. (16) approaches infinitely large (i.e., the sound power output of the system with active control is zero) when the surface interval $D$ is less than $0.5 \lambda$. This is because only the zero-th mode in the direction of the $z$-axis is available in the space. The cylindrical wave fronts that originated from both the primary source and the secondary source are perpendicular to the two reflecting surfaces, and they are completely suppressed with each other when the source strengths of the primary and secondary sources 
are the same magnitude but in anti-phase. The sound power output is complicated when the surface distance $D$ is larger than $0.5 \lambda$ due to the propagating of high order modes in the space.

For the infinitely large surfaces, it has been shown that the optimal configuration is that the source line is perpendicular to the two parallel surfaces. Therefore, this configuration is adopted for the case with finite size surfaces. For finite size surfaces, it is hard to obtain an analytical solution, and the sound field has to be solved using numerical methods. Although many kinds of software can be used to solve this model, this paper adopts the BEM in commercial software SYSNOISE, where both sides of reflecting surfaces are set as rigid boundaries. ${ }^{17}$ After the sound pressure at the positions of the primary and secondary sources is calculated, the self-radiation resistances of the primary and secondary sources and the mutual radiation resistance between them can be obtained similarly according to Eqs. (3)-(5) for the cases with finite size reflecting surfaces. The total sound power under the optimal active noise control is then obtained by Eq. (7).

\section{SIMULATIONS}

In this section, the source interval, $d$, is set to $0.1 \mathrm{~m}$ throughout the simulations, and the frequency of interest ranges from $315 \mathrm{~Hz}$ to $5 \mathrm{kHz}$ at the commonly used onethird octave center frequencies. The results with infinitely large reflecting surfaces are simulated and analyzed first and then are extended to the cases with finite size ones.

There are many different geometric configurations for the primary and secondary sources shown in Fig. 1. From Eqs. (12)-(14), it is clear that the noise reduction does not change with the distance between the midpoint of the source line and the reflecting surface for the major case $k D<\pi$. Therefore, only the configuration where the midpoint of the source line is on the middle line of the reflecting surfaces is investigated in the simulations for simplicity. The included angle $\theta$ between the source line and the normal line of reflecting surfaces ranges only from $0^{\circ}$ to $90^{\circ}$ thanks to the symmetry of the configuration. The surface interval $D$ is set to $0.1 \mathrm{~m}$, and the results are shown in Fig. 2. It is clear that the noise reduction is significantly affected by the included angle. At the frequencies below $1715 \mathrm{~Hz}$, which is the corresponding frequency when the surface interval equals the half wavelength, the system with a smaller included angle has higher noise reduction. For example, the noise reduction at $500 \mathrm{~Hz}$ increases from $7.8 \mathrm{~dB}$ to $19.6 \mathrm{~dB}$ when the angle decreases from $20^{\circ}$ to $5^{\circ}$.

The noise reduction performance is sensitive to the included angle. For example, the noise reduction is infinitely large when $\theta=0$ at $500 \mathrm{~Hz}$ but reduces to finite large $(19.6 \mathrm{~dB})$ when $\theta$ only increases by $5^{\circ}$, though it is still much larger than the noise reduction when there is no vertically placed reflecting surface $(6.0 \mathrm{~dB})$. It should be noted that the noise reduction performance with the parallel reflecting surfaces can be worse than that without the

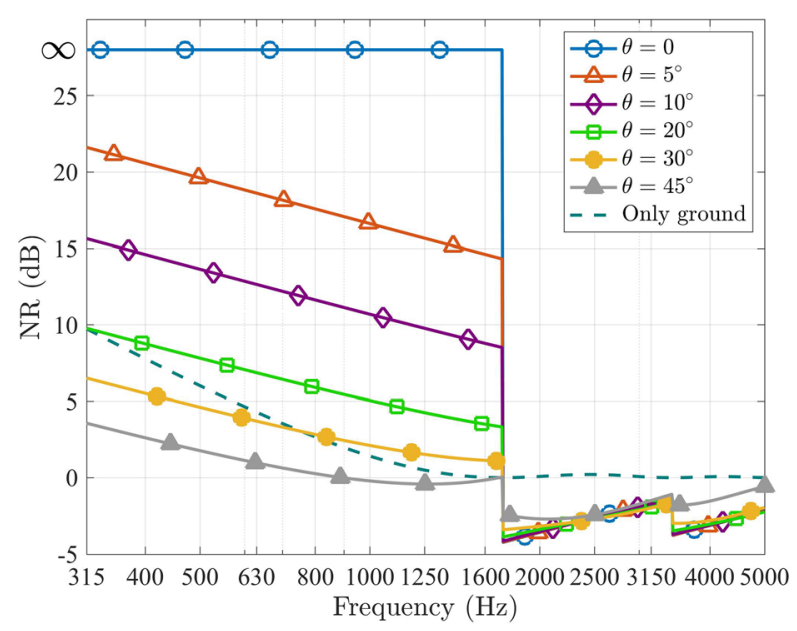

FIG. 2. (Color online) Noise reduction of the ANC system on ground with two parallel reflecting surfaces and with different included angles $\theta$ between the source line and the normal line of the surfaces.

surfaces (only ground) if the angle is large at low frequencies. For example, the noise reduction without the surface is $6.0 \mathrm{~dB}$ at $500 \mathrm{~Hz}$, but it decreases to $1.8 \mathrm{~dB}$ if the included angle is $45^{\circ}$.

There is a critical angle at which the noise reduction performance of the ANC system with the parallel reflecting surfaces is worse than without any placed surfaces. Figure 2 shows that the critical angle is about $20^{\circ}$ at low frequencies because the curve of " $\theta=20^{\circ}$ " intersects the one of "Only ground." This value of the critical angle can be obtained analytically. Applying Taylor series expansion of the Bessel function $\mathrm{J}_{0}(\cdot),{ }^{20}$ the noise reduction of the system with the surfaces described in Eq. (13) becomes

$$
\begin{aligned}
\mathrm{NR}= & -10 \lg \left(\frac { \pi } { k D } \left[\frac{1}{2}(k d \sin \theta)^{2}-\frac{2}{32}(k d \sin \theta)^{4}\right.\right. \\
& \left.\left.+o\left((k d \sin \theta)^{4}\right)\right]\right)
\end{aligned}
$$

where $o(\cdot)$ means the order less than the variables inside it. The noise reduction of the system without any reflecting surfaces described in Eq. (9) can then be expanded as ${ }^{21}$

$$
\mathrm{NR}_{\text {gnd }}=-10 \lg \left[\frac{1}{3}(k d)^{2}-\frac{2}{45}(k d)^{4}+o\left((k d)^{4}\right)\right] .
$$

Let Eq. (17) equal Eq. (18) and omit the order higher than $(k d)^{2}$, so the critical included angle $\theta_{c}$ is

$$
\theta_{\mathrm{c}}=\arcsin \sqrt{\frac{2 k D}{3 \pi}}
$$

Equation (19) shows that the critical angle does not depend on the source interval $d$ provided that the interval is small compared with the wavelength. For example, at $f=315 \mathrm{~Hz}$ and $D=0.1 \mathrm{~m}$, the value obtained from Eq. (19) is approximately $20.5^{\circ}$, which is consistent with that shown in Fig. 2. 


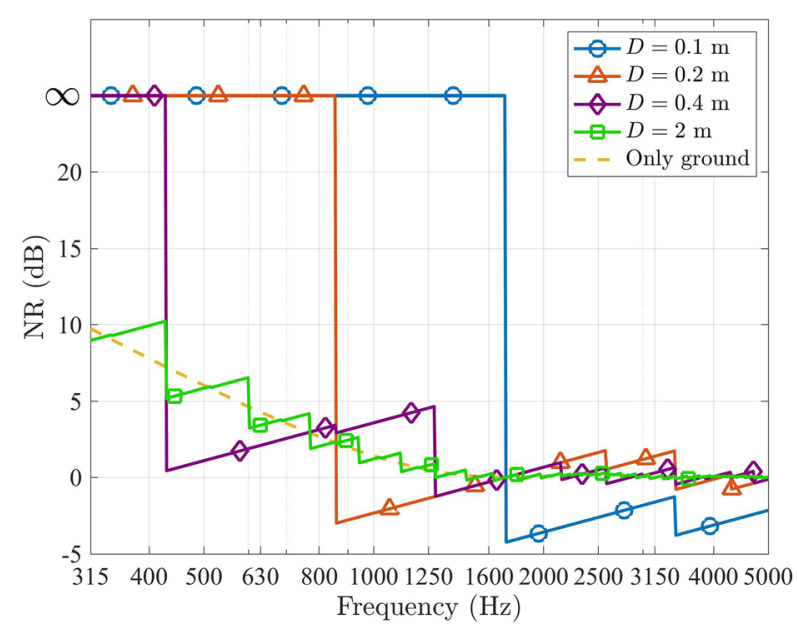

FIG. 3. (Color online) Noise reduction of an ANC system on ground with two parallel reflecting surfaces and the source line perpendicular to the surfaces at different surface intervals $D$, where the source interval $d=0.1 \mathrm{~m}$.

Figure 2 also shows that the noise reduction can be negative at the frequency larger than $1715 \mathrm{~Hz}$. The increase of the sound radiation power of the system is caused by the increase of the self-radiation resistance of the primary source from the parallel reflecting surfaces. Focusing on the optimal included angle $\theta=0$, Fig. 3 shows the effects of the surface interval $D$ on the noise reduction of the ANC system with the source distance $d$ being $0.1 \mathrm{~m}$. The corresponding turning frequency, which is defined as the frequency where the noise reduction drops from an infinitely large value down to a finite one, is 1715,858 , and $429 \mathrm{~Hz}$ for the source interval $D$ being $0.1,0.2$, and $0.4 \mathrm{~m}$, respectively.

These turning frequencies are equal to the values calculated by $c_{0} /(2 D)$ where $c_{0}$ is the sound speed in the air. As shown in Fig. 3, the turning frequency decreases as the surface interval increases, indicating that the effective frequency range for noise reduction improvement becomes lower with a larger surface interval. For example, when the surface interval is $2 \mathrm{~m}$, the noise reduction improvement only happens below $85.8 \mathrm{~Hz}$. After this turning frequency, the noise reduction curve in Fig. 3 fluctuates around the one without any surfaces. When the interval is much larger than $2 \mathrm{~m}$, the curve will approach to that without any surfaces because the surfaces are far away from the ANC systems and the effects of them become negligible. Therefore, to have effects at high frequencies, the two reflecting surfaces placed vertically in parallel need to be close to each other.

To illustrate why the noise reduction is infinitely large below the turning frequency, the secondary source strength $q_{\mathrm{s}, \mathrm{opt}}$ and the self-radiation resistances of two sources and their mutual-radiation resistance are shown in Fig. 4 for the configuration with $D=0.1 \mathrm{~m}$. When the frequency is less than $1715 \mathrm{~Hz}$, the secondary source strength is exactly opposite to that of the primary source and the mutual radiation resistance between sources $R_{\mathrm{ps}}$ and the self-radiation resistance of the secondary source $R_{\mathrm{s}}$ are exactly the same as shown in Fig. 4. The radiation caused by the resistances at the primary (or secondary) source is zero, indicating the overall sound power of the total system is zero. This result is consistent with the analytical analyses in Sec. II.

At the frequencies larger than $1715 \mathrm{~Hz}$, the noise reduction with two reflecting surfaces is negative, indicating that the sound power of the system increases. Figure 4(b) also shows that the value of the self-radiation resistance of the primary source $R_{\mathrm{p}}$ jumps discontinuity from $R_{0}$ to $3 R_{0}$ at $1715 \mathrm{~Hz}$ due to the perfect reflection of the reflecting surfaces, ${ }^{14}$ resulting in a $4.8 \mathrm{~dB}$ increase of the sound power of the primary source. When the secondary source is introduced, there is a $0.4 \mathrm{~dB}$ reduction, as calculated by using Eqs. (10) and (11), so the noise reduction is $-4.4 \mathrm{~dB}$. The secondary source in this case has little effect on the primary source because the source interval is larger than the half wavelength. At higher frequencies, the noise reduction of the ANC system tends to $-3.0 \mathrm{~dB}$. This is because the

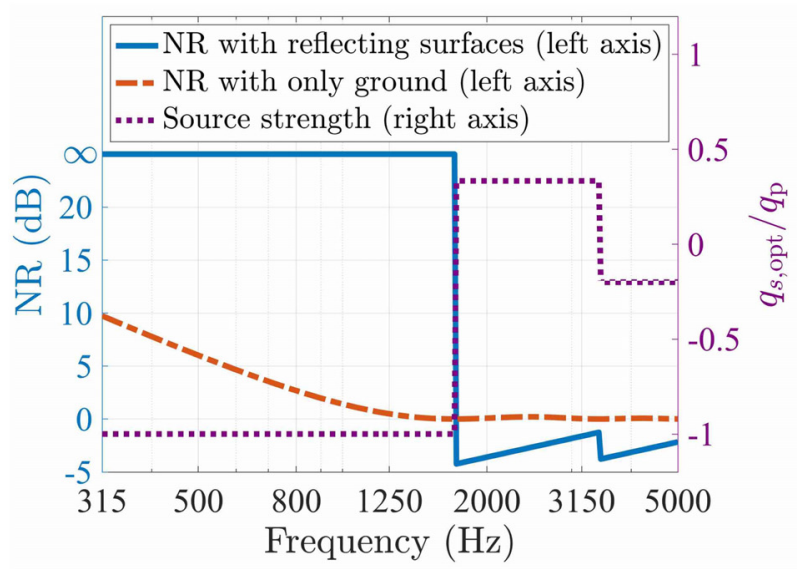

(a)

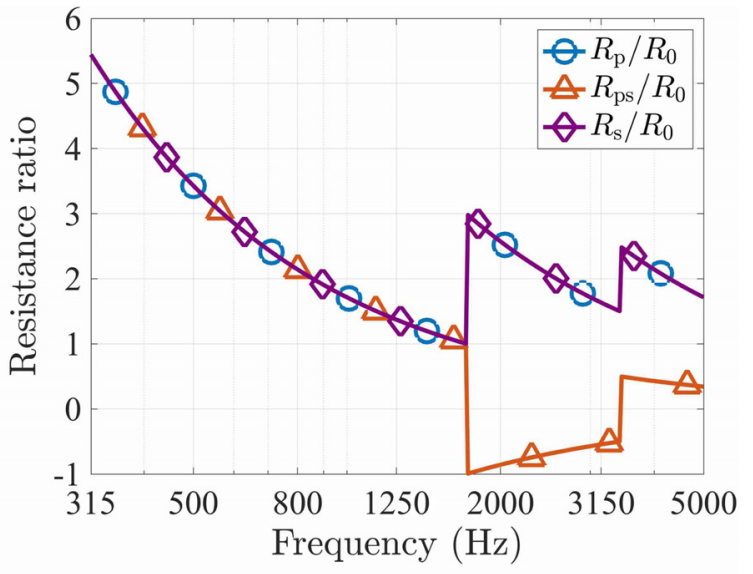

(b)

FIG. 4. (Color online) Two infinitely large parallel reflecting surfaces are introduced to a single channel ANC system on ground: (a) noise reduction (left $y$ axis) and the ratio of the source strengths $q_{\mathrm{s}}$,opt $/ q_{\mathrm{p}}$, (right $y$ axis) where $q_{\mathrm{s} \text {,opt }}$ and $q_{\mathrm{p}}$ is the source strength of the secondary and the primary source, respectively; (b) the self-radiation resistances of the primary and secondary sources, $R_{\mathrm{p}}$ and $R_{\mathrm{s}}$, and the mutual radiation resistance between the two sources $R_{\mathrm{ps}}$, where $R_{0}=\left(\rho_{\text {air }} \omega k\right) /(2 \pi)$ is the self-radiation resistance of the primary source on ground without any vertically placed reflecting surfaces. 
reflecting surface close to the primary source doubles its self-radiation resistance and the effect of the secondary source becomes negligible when the distance between the sources is larger than a wavelength.

In practical applications, the reflecting surfaces cannot be infinitely large. To focus on the effects of the finite size reflecting surfaces, the source line is set to be perpendicular to the surfaces. The surface interval $D$ is set to $0.1 \mathrm{~m}$, so the turning frequency is approximately $1715 \mathrm{~Hz}$ for the case of infinitely large surfaces, as analyzed previously. The source distance is set to $0.08 \mathrm{~m}$, and the distance between the source and its nearer reflecting surface is $0.01 \mathrm{~m}$ to avoid computation errors. The ratio of the width $w$ to the height $h$ of each surface is set to 1 , and the sources are located on the perpendicular bisector of the line intersected by the surfaces and the ground plane.

The noise reduction of the ANC system with finite size reflecting surfaces is shown in Fig. 5, where the noise reduction below $1715 \mathrm{~Hz}$ increases with the size of the reflecting surfaces. For example, at $315 \mathrm{~Hz}$, there is about $5-6 \mathrm{~dB}$ increment in the noise reduction of the ANC system for each $0.05 \mathrm{~m}$ increment in the side length of the square reflecting surfaces. The increment is more significantly in the middle frequency range, such as around $1 \mathrm{kHz}$, where the noise reduction of the configuration with a size of $0.2 \mathrm{~m}$ is $10.4 \mathrm{~dB}$ more than that of the one with a size of $0.15 \mathrm{~m}$. Therefore, the finite size reflecting surfaces can be used to increase the noise reduction performance of the ANC system on ground below the turning frequency, whose corresponding half wavelength is equal to the surface interval.

At the frequencies below $315 \mathrm{~Hz}$, further simulations (not presented in this paper) show that the noise reduction of the ANC system is still infinitely large for the infinitely large reflecting surfaces but increases by about $6 \mathrm{~dB}$ as the frequency is halved for the cases with and without finite size ones. Although the radiation of each source with finite size reflecting surfaces approaches to that without any reflecting surfaces at low frequencies, the noise reduction with reflecting surfaces is still larger than that without any reflecting

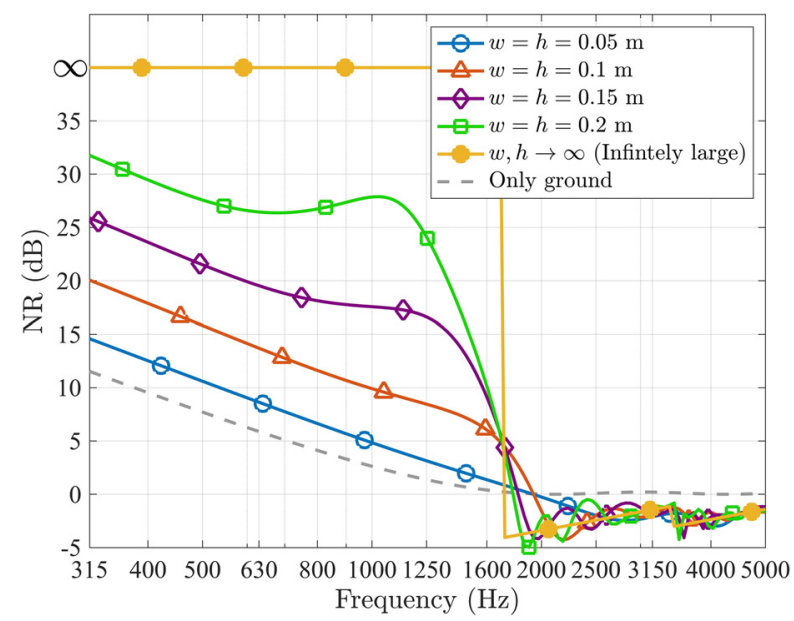

FIG. 5. (Color online) Noise reduction of the ANC system on ground with two parallel reflecting surfaces, where the surface interval $D=0.1 \mathrm{~m}$. surfaces because the surface size is still comparable with respect to the source distance and affects their mutual radiation. Therefore, introducing reflecting surfaces is always beneficial at low frequencies.

\section{EXPERIMENTS}

The experiments with a single channel ANC system were conducted in a full anechoic room in Nanjing University with dimensions of $11.4 \mathrm{~m} \times 7.8 \mathrm{~m} \times 6.7 \mathrm{~m}$. The sketch and photographs of experimental setups are shown in Figs. 6 and 7, respectively. The infinitely large ground was approximated by a $6 \mathrm{~m} \times 6 \mathrm{~m}$ wooden plate, and two $1.2 \mathrm{~m} \times 1.2 \mathrm{~m}$ wooden plates were used to approximate the two infinitely large reflecting surfaces placed vertically on ground in parallel. Three sets of finite size surfaces, $0.1 \mathrm{~m} \times 0.1 \mathrm{~m}, 0.15 \mathrm{~m} \times 0.15 \mathrm{~m}$, and $0.2 \mathrm{~m} \times 0.2 \mathrm{~m}$, were tested at different frequencies. All of the wooden plates used in the experiments have a thickness of $1.8 \mathrm{~cm}$ and a surface density of $15.30 \mathrm{~kg} / \mathrm{m}^{2}$. The ratio of the sound power reflected from the wooden plate to the total sound power radiated from the sound source is larger than $96.6 \%$ above $100 \mathrm{~Hz}$, so this setup can approximate the rigid surface condition. $^{22}$

The sound power of the single channel ANC system was measured with 10 measurement microphones located on a semispherical surface centered at the source with the radius of $2.5 \mathrm{~m}$, as shown in Fig. 7(a) according to the 10 positions listed in ISO $3744 .{ }^{23}$ The sound pressure at the measurement microphones was sampled with a Brüel \& Kjær PULSE 3560 D Analyzer (Skodsborgvej 307, 2850 Nærum, Denmark) and the fast Fourier transform (FFT) analyzer in PULSE LabShop was used to obtain the FFT spectrum. Both primary and secondary sources are customized loudspeakers, and each one was made by assembling a 1inch loudspeaker unit in a $48 \mathrm{~mm}$ (length) $\times 48 \mathrm{~mm}$ (width) $\times 38 \mathrm{~mm}$ (depth) plexiglass box. The acoustic center of such a loudspeaker was considered at the geometric center of the diaphragm of the loudspeaker.

A commercial active noise controller (Antysound Tiger ANC WIFI-M, 20-203 Guangzhou Rd., Nanjing, China) embedded with the waveform synthesis algorithm was used for control. ${ }^{24}$ The internally synthesized signal at preset frequencies was used to drive the primary source and adopted as the reference signal. Considering the frequency response of the loudspeakers and the computation capability of the controller, the experiments were conducted at a number of pure tones from $300 \mathrm{~Hz}$ to $2 \mathrm{kHz}$ with an interval of $50 \mathrm{~Hz}$.

The objective of this research is to minimize the sound power of the system. In the theories and simulations, the minimized sound power of two sources under the optimal control can be calculated directly according to Eq. (7). In the experiments, the minimized sound power can be achieved by feeding the pre-calculated optimal signal into the secondary source if the transfer functions of the system and the source strength of the primary source are fixed and can be obtained in advance by simulations or measurements. 


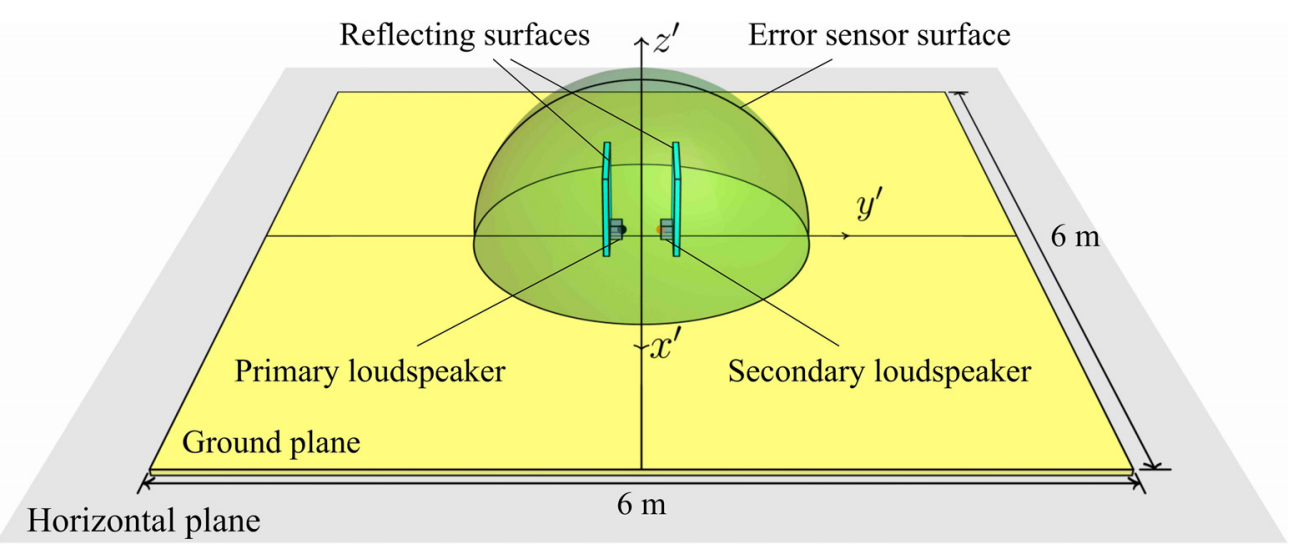

FIG. 6. (Color online) Sketch of the experimental setup.

However, the characteristics of the noise source, the acoustic environment, and the secondary path transfer functions from the secondary source to the error sensors might vary during the experiments period, so the noise reduction

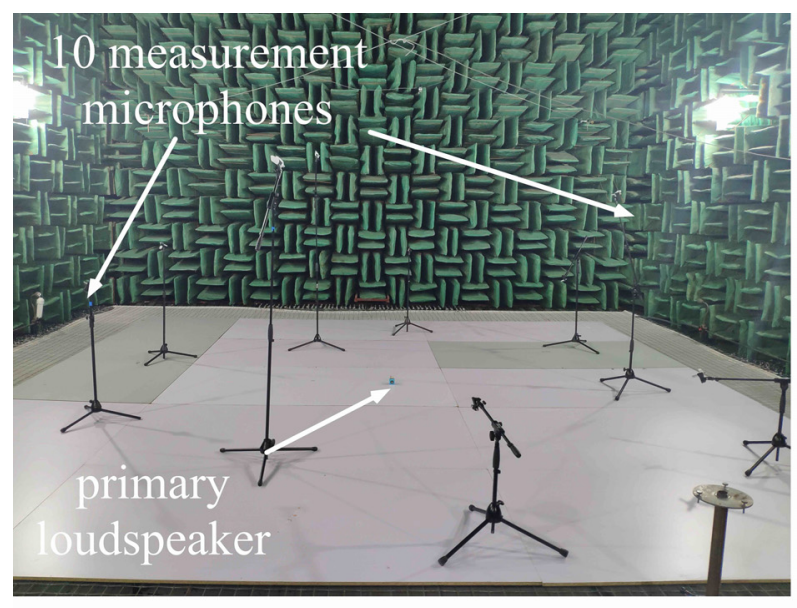

(a)

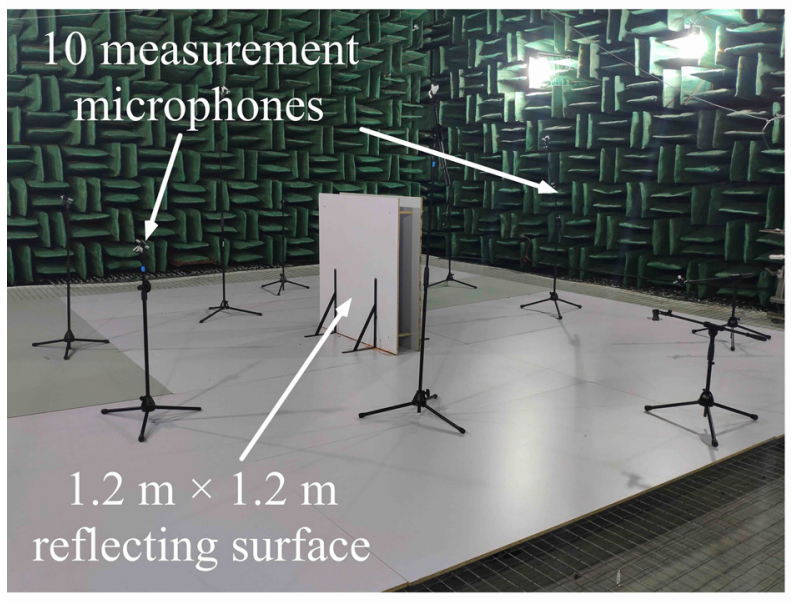

(c) performance obtained with the pre-calculated optimal control signal might not be good. To overcome the difficulty, adaptive control was adopted, which could track these variations and achieved optimal noise reduction performance in

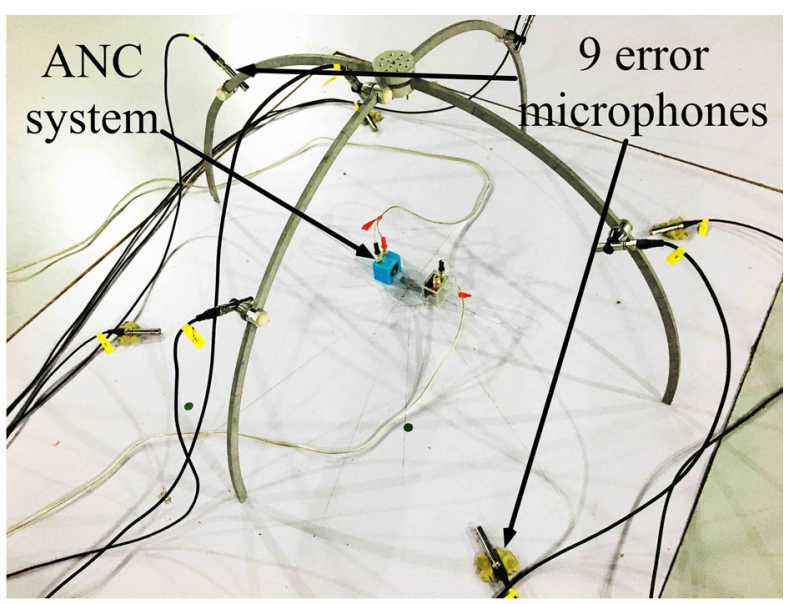

(b)

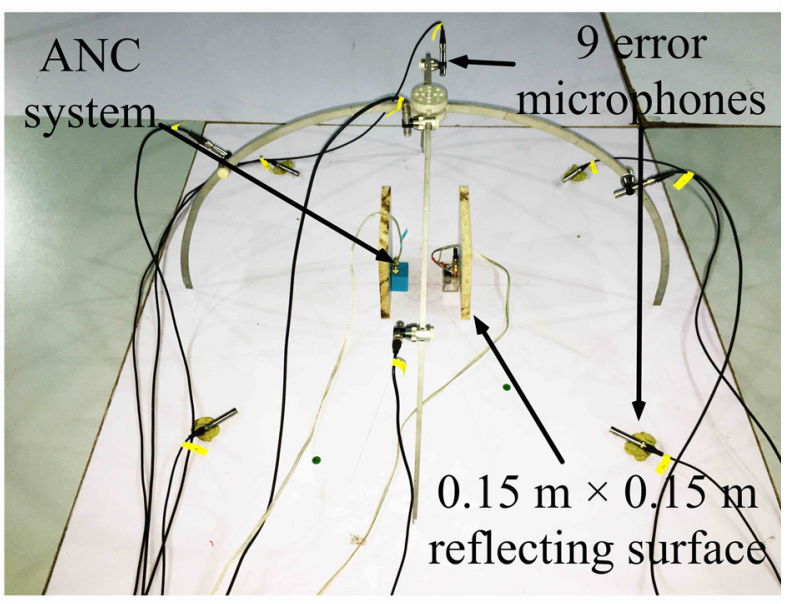

(d)

FIG. 7. (Color online) Photographs of the experimental setup: (a) the ground plane approximated by a $6 \mathrm{~m} \times 6 \mathrm{~m}$ wooden plate and the $10 \mathrm{measurement}$ microphones; (b) a single channel ANC system on ground with 9 error microphones; (c) a single channel ANC system on ground with two vertically placed reflecting surfaces $(1.2 \mathrm{~m} \times 1.2 \mathrm{~m})$; (d) a single channel ANC system on ground with two vertically placed reflecting surfaces $(0.15 \mathrm{~m} \times 0.15 \mathrm{~m})$ and 9 error microphones. 
the experiments. In the adaptive control system, the summation of the squared sound pressure at error microphones is minimized because it is a good approximation of the sound power of the system, which cannot be measured directly.

The sound power noise reduction obtained in the experiment $\left(\mathrm{NR}_{\mathrm{p}}\right)$ is less than $\mathrm{NR}_{\mathrm{w}}$, which is obtained by minimizing the sound power theoretically. For the case without additional reflecting surfaces (only ground) and the case with two finite size reflecting surfaces, the number and optimal locations of the error microphones obtained by the simulations are given in Table I, where the zenith angle and the azimuth angle are the angular coordinates in the spherical coordinate system transformed from the Cartesian one $\left[x^{\prime}, y^{\prime}, z^{\prime}\right]$ shown in Fig. 6. These locations are similar to the ones specified by Table B.2 of the sound power measurement standard ISO $3744 .^{23}$ The summation of the squared sound pressure at these error microphones is approximately proportional to the sound power. Therefore, minimizing the summation is equivalent to minimizing the radiated sound power of the system.

A semispherical support frame with a radius of $0.5 \mathrm{~m}$ centered at the midpoint of the two sources, which served as the error sensor surface shown in Fig. 6, was used to install the 9 error microphones. For the case with two infinitely large parallel reflecting surfaces (approximated by two $1.2 \mathrm{~m} \times 1.2 \mathrm{~m}$ wooden plates), it is inconvenient to install the semispherical support frame as in the former cases. Only the error microphones, which are located in the middle plane of the two reflecting surfaces (i.e., the 1st, 2 nd, and 5 th ones in Table I), are installed by microphone stands.

The simulations (not presented in this paper) by the authors show that the difference between the sound power reduction obtained by minimizing the sum of the squared sound pressure at these error microphones and the one by minimizing the sound power theoretically is less than $0.2 \mathrm{~dB}$ in the frequency range from $300 \mathrm{~Hz}$ to $1.4 \mathrm{kHz}$. It means controlling the sound pressure at error microphones can achieve the same noise reduction as minimizing the total sound power. The simulation results (not presented in this paper) shown that the radiation pattern of the system under the optimal control is not uniform. The noise reduction is the largest in the direction above the midpoint of the source line. Increasing the size of the reflecting surfaces can improve the noise reduction performance at all directions, but the radiation directivity pattern remains similar.

The measured noise reduction, defined as the measured sound power level with the two parallel reflecting surfaces under optimal control subtracted from the one without the reflecting surfaces (only ground) and without ANC, is

TABLE I. Locations of the error microphones in the experiments for the case without additional reflecting surfaces (only ground) and the case with two finite size parallel reflecting surfaces.

\begin{tabular}{lccccccccc}
\hline \hline No. of the error mic. $i$ & 1 & 2 & 3 & 4 & 5 & 6 & 7 & 8 & 9 \\
\hline Zenith angle $\left(^{\circ}\right)$ & 0 & 45 & 45 & 45 & 45 & 90 & 90 & 90 & 90 \\
Azimuth angle $\left(^{\circ}\right)$ & 0 & 0 & 90 & 180 & 270 & 45 & 135 & 225 & 315 \\
\hline
\end{tabular}

shown in Fig. 8 at different included angles. In this configuration, the surface interval $D$ is set to $0.2 \mathrm{~m}$ for easy installation of the $1.2 \mathrm{~m} \times 1.2 \mathrm{~m}$ surfaces, and the source interval $d$ is set to $0.074 \mathrm{~m}$, which is consistent with all of the configurations in the experiments. It can be found in Fig. 8 that the noise reduction decreases significantly as the included angle increases, which is well predicted by the simulations. The measured result at $0^{\circ}$ is only $22.9 \mathrm{~dB}$, which is a finite value instead of the infinitely large one, as predicted. This might be due to the fact that the actual parallel reflecting surfaces are not large enough and the existence of background noise in the control system.

The measured noise reduction at different surface intervals with two reflecting surfaces placed vertically in parallel is shown in Fig. 9. The turning frequencies from large noise reductions down to small ones are about $600 \mathrm{~Hz}$ and $1150 \mathrm{~Hz}$ for the case $D=0.15 \mathrm{~m}$ and $0.3 \mathrm{~m}$, respectively, which is close to the predicted values of $572 \mathrm{~Hz}$ and $1143 \mathrm{~Hz}$. The noise reductions below the turning frequency are between $20 \mathrm{~dB}$ and $25 \mathrm{~dB}$, which are about $10 \mathrm{~dB}$ higher than those without any surfaces (only ground). It is clear that the noise reduction of an ANC system on ground can be highly improved by introducing two parallel reflecting surfaces close to sources.

The noise reductions above $600 \mathrm{~Hz}$ for the case $D=0.3 \mathrm{~m}$ is higher than those from the simulations. Some reasons include the following. First, it was found in the experiments that the sound reduction performance is sensitive to the parallel degree of the two reflecting surfaces, but it was hard to make them be perfectly parallel to each other due to the flexible floor on the mesh of the anechoic chamber. Second, it is assumed that the sources are point monopole in the theory and simulations, but the loudspeakers used in the experiments are not omnidirectional in the middle and high frequency range. Last, it was hard to place the two loudspeakers accurately at the required locations between

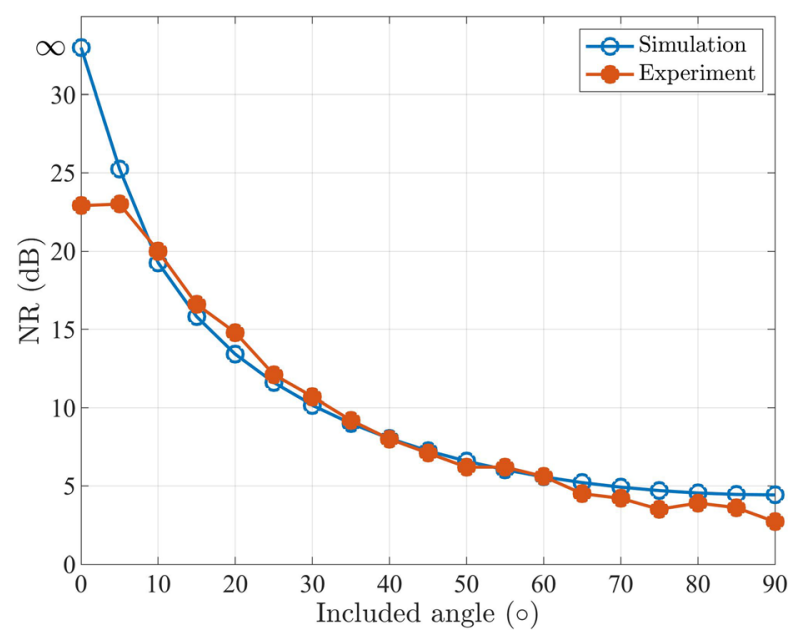

FIG. 8. (Color online) Comparisons of the noise reduction of the ANC system at $500 \mathrm{~Hz}$ with two reflecting surfaces, which are placed vertically in parallel at different included angles between the source line and the normal line of the surfaces. The surface interval $D=0.2 \mathrm{~m}$ and the source interval $d=0.074 \mathrm{~m}$. 


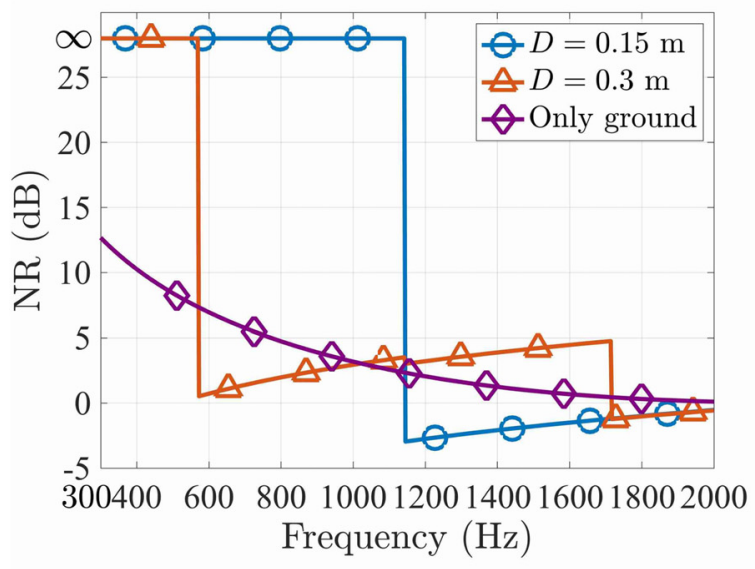

(a)

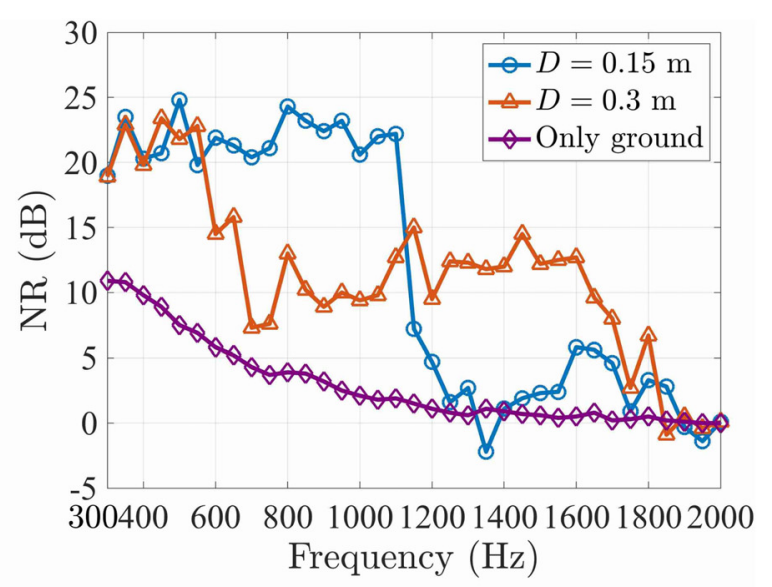

(b)

FIG. 9. (Color online) Comparisons of the noise reduction of the ANC system with two reflecting surfaces, which are placed vertically in parallel at different surface intervals, where the source interval $d=0.074 \mathrm{~m}$ : (a) the simulations and (b) the experiments.

reflecting surfaces, and the actual separation of acoustic centers of loudspeakers might be less than $0.074 \mathrm{~m}$.

The measured noise reduction with three sets of finite size surfaces, $0.1 \mathrm{~m} \times 0.1 \mathrm{~m}, 0.15 \mathrm{~m} \times 0.15 \mathrm{~m}$, and $0.2 \mathrm{~m} \times 0.2 \mathrm{~m}$, at different frequencies is shown in Fig. 10. In the experiments, the surface interval $D$ is $0.15 \mathrm{~m}$, and the loudspeakers were placed close to the adjacent surface, so the source interval $d$ is about $0.074 \mathrm{~m}$. The experimental results are generally in accordance with the simulation ones. So finite size reflecting surfaces that are placed vertically on ground in parallel can improve the noise reduction performance of the ANC system, and better noise reduction can be achieved with larger size reflecting surfaces at low frequencies. For example, in the experiments, the noise reduction improvement of that without any surfaces (only ground) at $800 \mathrm{~Hz}$ is $2.5 \mathrm{~dB}(2.8 \mathrm{~dB}$ in the simulation) for the configuration $0.1 \mathrm{~m} \times 0.1 \mathrm{~m}$, and increases to $8.6 \mathrm{~dB}(11.9 \mathrm{~dB}$ in the simulation) for the configuration $0.2 \mathrm{~m} \times 0.2 \mathrm{~m}$.

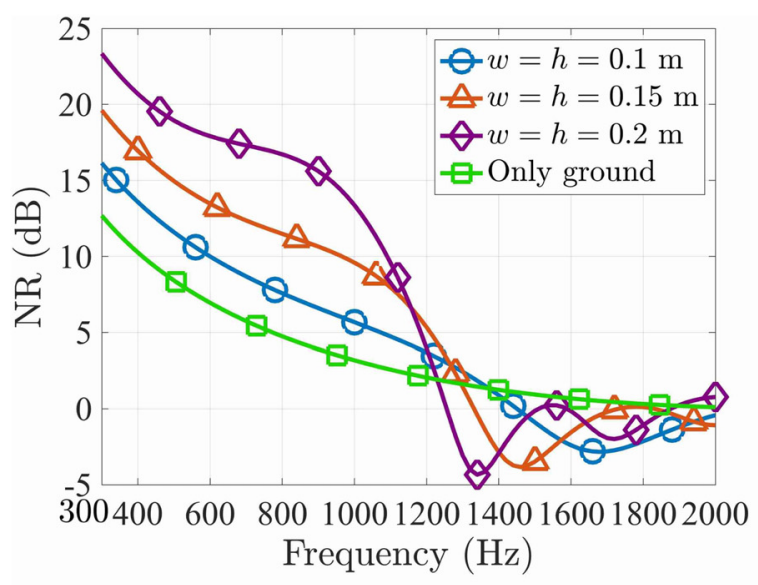

(a)
The noise reductions for the different configurations mentioned above are summarized in Table II at two typical frequencies of $500 \mathrm{~Hz}$ and $800 \mathrm{~Hz}$. The global control of the noise source on ground without any additional reflecting surfaces is limited, and all the NRs are less than $10 \mathrm{~dB}$. However, the NRs are greater than $10 \mathrm{~dB}$ in both the simulations and experiments if the size of surfaces is greater than $0.1 \mathrm{~m} \times 0.1 \mathrm{~m}$ at $500 \mathrm{~Hz}$ and $0.2 \mathrm{~m} \times 0.2 \mathrm{~m}$ at $800 \mathrm{~Hz}$. Therefore, it is feasible to improve the NR of the ANC system by introducing two parallel reflecting surfaces with proper distance and size.

\section{CONCLUSIONS}

This paper employs the modal expansion method and the boundary element method to simulate the noise reduction of ANC systems on ground with infinitely large and finite size reflecting surfaces that are placed vertically on

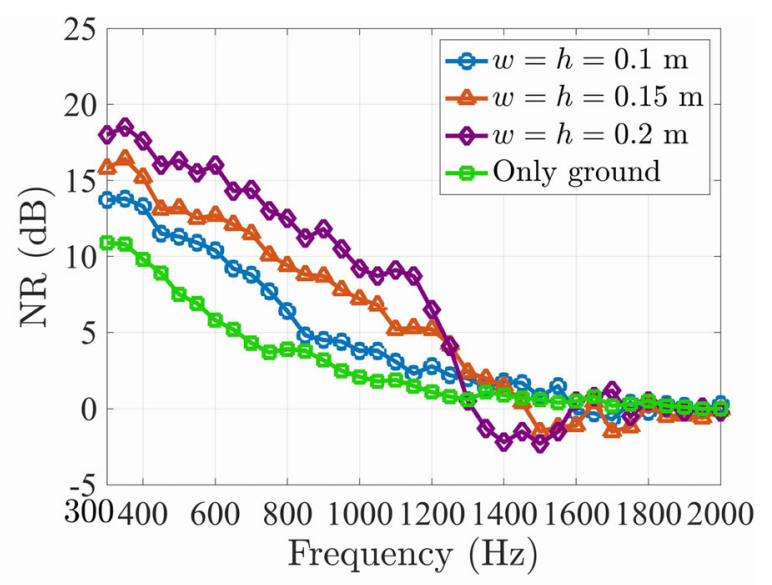

(b)

FIG. 10. (Color online) Noise reduction of the ANC system with two finite size and parallel reflecting surfaces where the surface interval $D=0.15 \mathrm{~m}$ and the source interval $d=0.074 \mathrm{~m}$ : (a) the simulations and (b) the experiments. 
TABLE II. The noise reductions (NRs) when the surface interval $D=0.15 \mathrm{~m}$ and the source interval $d=0.074 \mathrm{~m}$.

\begin{tabular}{|c|c|c|c|c|c|}
\hline \multicolumn{2}{|l|}{ Configurations } & \multicolumn{2}{|c|}{$500 \mathrm{~Hz}$} & \multicolumn{2}{|c|}{$800 \mathrm{~Hz}$} \\
\hline & Size & Simulation & Experiment & Simulation & Experiment \\
\hline Only ground & $\backslash$ & $8.4 \mathrm{~dB}$ & $7.5 \mathrm{~dB}$ & $4.8 \mathrm{~dB}$ & $3.9 \mathrm{~dB}$ \\
\hline \multirow[t]{4}{*}{ With two reflecting surfaces } & $0.1 \mathrm{~m} \times 0.1 \mathrm{~m}$ & $11.6 \mathrm{~dB}$ & $11.3 \mathrm{~dB}$ & $7.6 \mathrm{~dB}$ & $6.4 \mathrm{~dB}$ \\
\hline & $0.15 \mathrm{~m} \times 0.15 \mathrm{~m}$ & $15.0 \mathrm{~dB}$ & $13.2 \mathrm{~dB}$ & $11.5 \mathrm{~dB}$ & $9.4 \mathrm{~dB}$ \\
\hline & $0.2 \mathrm{~m} \times 0.2 \mathrm{~m}$ & $18.9 \mathrm{~dB}$ & $16.3 \mathrm{~dB}$ & $16.7 \mathrm{~dB}$ & $12.5 \mathrm{~dB}$ \\
\hline & Infinitely large & $\infty$ & $24.8 \mathrm{~dB}$ & $\infty$ & $24.3 \mathrm{~dB}$ \\
\hline
\end{tabular}

ground around the system in parallel. Both experimental and simulation results demonstrate that the system noise reduction performance can be improved significantly with the introduction of the two reflecting surfaces. The optimal performance occurs when the source line is perpendicular to the two parallel surfaces. With infinitely large reflecting surfaces, the sound radiation of the primary source can be completely suppressed in principle by a secondary source, provided that the surface distance is less than the half wavelength. With finite size reflecting surfaces, the noise reduction performance improvement is still significant. For example, for an ANC system with the source distance of $0.074 \mathrm{~m}$ on ground, the original noise reduction is $3.9 \mathrm{~dB}$ at $800 \mathrm{~Hz}$. After vertically placing two $0.2 \mathrm{~m} \times 0.2 \mathrm{~m}$ reflecting surfaces around the system in parallel, the noise reduction is $12.5 \mathrm{~dB}$, which is $8.6 \mathrm{~dB}$ higher than without any reflecting surfaces. Future research includes exploring the optimal geometrical shape of the finite size reflecting surfaces and optimal configurations of the error sensors and secondary sources for multiple channel ANC systems with two parallel reflecting surfaces.

\section{ACKNOWLEDGMENTS}

This research was supported by the National Science Foundation of China (Grant Nos. 11874218 and 11874219).

\section{APPENDIX}

This appendix presents the derivation of Eqs. (10) and (11). When using Eqs. (3)-(5) to calculate the self-radiation and mutual radiation resistances of the primary and secondary sources, the $z$-axis of the cylindrical coordinate system $(\rho, \varphi, z)$ can be established at a line going through the primary or the secondary source and perpendicular to the reflecting surfaces. In such a case, the radial coordinate $\rho_{<}$is always 0 in Eq. (1). Then Eq. (1) can be simplified by using the special values of the Bessel functions, $\mathrm{J}_{n}(0)=1$ for $n=0$ and $\mathrm{J}_{n}(0)=0$ for $n=1,2,3, \ldots$, as

$$
\begin{aligned}
p\left(\mathbf{r} ; \mathbf{r}_{0}\right)= & \frac{\rho_{\mathrm{air}} \omega k q_{\mathrm{src}}}{4 \pi} \sum_{n=0}^{\infty} \frac{\pi}{k D} \varepsilon_{n} \cos \left(\frac{z}{D} n \pi\right) \\
& \times \cos \left(\frac{z_{0}}{D} n \pi\right) \mathrm{H}_{0}\left(\sqrt{1-\left(\frac{n \pi}{k D}\right)^{2}} k \rho_{>}\right) .
\end{aligned}
$$

It is found that the argument of the Hankel function of the first kind $\mathrm{H}_{0}(\cdot)$ is a non-negative real number and a pure imaginary one when $k D \geq n \pi$ and $k D<n \pi$, respectively. When the argument is non-negative, the results of the Hankel function are well known as $\mathrm{H}_{0}(x)=\mathrm{J}_{0}(x)+\mathrm{j} \mathrm{Y}_{0}(x)$, where $\mathrm{J}_{0}(x)$ and $\mathrm{Y}_{0}(x)$ are the Bessel functions of the first and second kind, respectively. Substituting this relationship into the Eqs. (3) to (5) for the resistance calculation, only the real part of the $\mathrm{H}_{0}(x)$ [i.e., the $\mathrm{J}_{0}(x)$ ] remains.

When the argument is a pure imaginary number $\mathrm{j} x$, the Hankel function in Eq. (A1) can be formulated as (page 200 in Ref. 25)

$$
\mathrm{K}_{m}(x)=\frac{\pi}{2} \mathrm{j}^{m+1} \mathrm{H}_{m}(\mathrm{j} x),
$$

where $\mathrm{K}_{m}(x)$ is the modified Bessel function of the second kind and a real number if $m=0$ and $x$ are non-negative real numbers. ${ }^{20}$ Therefore, $\mathrm{H}_{0}(\mathrm{j} x)$ is a pure imaginary number, which means the real part is zero and has no contributions to the resistances computed by Eqs. (3)-(5). So, the summation terms in Eq. (A1) that satisfy $k D<n \pi$ are neglected, and the upper bound of the summation in Eqs. (10) and (11) is $\lfloor k D / \pi\rfloor$.

${ }^{1}$ S. Boodoo, R. Paurobally, and Y. Bissessur, "A review of the effect of reflective surfaces on power output of sound sources and on actively created quiet zones," Acta Acust. United Ac. 101(5), 877-891 (2015).

${ }^{2}$ K. A. Cunefare and S. Shepard, "The active control of point acoustic sources in a half-space,” J. Acoust. Soc. Am. 93(5), 2732-2739 (1993).

${ }^{3}$ W. S. Shepard, Jr. and K. A. Cunefare, "Active control of extended acoustic sources in a half-space," J. Acoust. Soc. Am. 96(4), 2262-2271 (1994).

${ }^{4}$ J. Tao, S. Wang, X. Qiu, and J. Pan, "Performance of a multichannel active sound radiation control system near a reflecting surface," Appl. Acoust. 123, 1-8 (2017).

${ }^{5}$ J. Pan, X. Qiu, and R. Paurobally, "Effect of reflecting surfaces on the performance of active noise control," in Proceedings of ACOUSTICS 2006, Christchurch, New Zealand (2006), pp. 69-74.

${ }^{6}$ J. Zhong, J. Tao, and X. Qiu, "Increasing the performance of active noise control systems on ground with a finite size vertical reflecting surface," Appl. Acoust. 154, 193-200 (2019).

${ }^{7}$ J. Xue, J. Tao, and X. Qiu, "Performance of an active control system near two reflecting surfaces," in Proceedings of 20th International Congress on Sound and Vibration, Bangkok, Thailand (2013).

${ }^{8}$ X. Qiu, J. Lu, and J. Pan, "A new era for applications of active noise control," in Proceedings of Inter-noise 2014, Melbourne, Australia (2014), pp. 1254-1263.

${ }^{9}$ J. Zhong, J. Tao, and X. Qiu, "Increasing the performance of active noise control systems on ground with two vertical reflecting surfaces with an included angle," J. Acoust. Soc. Am. 146(6), 4075-4085 (2019). 


\section{JASA}

${ }^{10} \mathrm{U}$. Ingard and G. L. Lamb, Jr., "Effect of a reflecting plane on the power output of sound sources," J. Acoust. Soc. Am. 29(6), 743-744 (1957).

${ }^{11}$ D. A. Bies, "Effect of a reflecting plane on an arbitrarily oriented multipole," J. Acoust. Soc. Am. 33(3), 286-288 (1961).

${ }^{12}$ F. Martinus, Z. Quabili, and A. Seybert, "Sound through partial enclosures with louvers," SAE Transactions, 1857-1862 (2001).

${ }^{13}$ B. Lam, C. Shi, D. Shi, and W.-S. Gan, "Active control of sound through full-sized open windows," Build. Environ. 141, 16-27 (2018).

${ }^{14}$ R. V. Waterhouse, "Output of a sound source in a reverberation chamber and other reflecting environments," J. Acoust. Soc. Am. 30(1), 4-13 (1958).

${ }^{15}$ R. V. Waterhouse, "Radiation impedance of a source near reflectors," J. Acoust. Soc. Am. 35(8), 1144-1151 (1963).

${ }^{16}$ L. M. Brekhovskikh, Waves in Layered Media (Academic Press, New York, 1980).

${ }^{17}$ SYSNOISE Users Manual for the Revision 5.6 (LMS International, Leuven, Belgium, 2003).

${ }^{18}$ S. J. Elliott, P. Joseph, P. Nelson, and M. Johnson, "Power output minimization and power absorption in the active control of sound," J. Acoust. Soc. Am. 90(5), 2501-2512 (1991).
${ }^{19}$ P. A. Nelson and S. J. Elliott, Active Control of Sound (Academic Press, San Diego, CA, 1992).

${ }^{20} \mathrm{~S}$. Zhang and J. Jin, Computation of Special Functions (John Wiley \& Sons, New York, 1996).

${ }^{21}$ P. Nelson, A. Curtis, S. Elliott, and A. Bullmore, "The minimum power output of free field point sources and the active control of sound," J. Sound Vib. 116(3), 397-414 (1987).

${ }^{22}$ K. Yamada, H. Takahashi, and R. Horiuchi, "Theoretical and experimental investigation of sound power transmitting through reflecting plane with low surface density in the calibration of reference sound sources," Acoust. Sci. Tech. 36(4), 374-376 (2015).

${ }^{23}$ ISO 3744:2010 Acoustics: Determination of sound power levels and sound energy levels of noise sources using sound pressure; Engineering methods for an essentially free field over a reflecting plane (International Organization for Standardization, Genève, Switzerland, 2010).

${ }^{24} \mathrm{X}$. Qiu and C. H. Hansen, "An algorithm for active control of transformer noise with on-line cancellation path modelling based on the perturbation method," J. Sound Vib. 240(4), 647-665 (2001).

${ }^{25}$ I. S. Gradshteyn and I. M. Ryzhik, Table of Integrals, Series, and Products (Academic Press, Waltham, MA, 2014). 